This document must be cited according to its final version which is published in a journal as:

M. Nadri ${ }^{1}$, H.Hammouri ${ }^{1}$, C. M.Astorga Zaragoza ${ }^{1}$

"Observer Design for Continuous-Discrete Time State Affine Systems up to Output Injection", European Journal of Control

10,03 (2004) 252-263

This final version may be found:

http://dx.doi.org/10.3166/ejc.10.252-263

All open archive documents of $\mathbf{H}$. Hammouri are available at: http://hal.archives-ouvertes.fr/HAMMOURI-HASSAN

All open archive documents of $\mathbf{H}$. Hammouri research group (SNLEP) are available at:

http://hal.archives-ouvertes.fr/SNELP http://www.tinyurl.com/SNELP

The professional web page (Fr/En) of $\mathrm{H}$. Hammouri is:

http://www.lagep.univ-lyon1.fr/signatures/hammouri.hassan

1

Université de Lyon, Lyon, F-69003, France; Université Lyon 1;

CNRS UMR 5007 LAGEP (Laboratoire d'Automatique et de GEnie des Procédés),

43 bd du 11 novembre, 69100 Villeurbanne, France

Tel +33 (0) 472431845 - Fax +33 (0) 472431699

http://www-lagep.univ-lyon1.fr/ http://www.univ-lyon1.fr http://www.cnrs.fr 
Article Submitted to ${ }^{* * *}$ European Journal of Control. ${ }^{* * *}$

\title{
Continuous-discrete time observer design for state affine systems up to output injection
}

\author{
M. NADRI ${ }^{\dagger}$, H. Hammouri ${ }^{\dagger *}$ AND C. Astorga ${ }^{\dagger}$, \\ ${ }^{\dagger}$ Laboratoire d'Automatique et de Génie des Procédés L.A.G.E.P., CNRS UMR 500\%, \\ Université Claude Bernard Lyon I, ESCPE - Lyon, Bât. 308 G, 43 Bd du 11 Novembre 1918, \\ 69622 Villeurbanne cedex, France.
}

${ }^{*}$ Corresponding author Tel. 334724318 95; Fax. 334724316 99; E-mail hammouri@lagep.cpe.fr 


\begin{abstract}
By continuous-discrete time affine systems, we mean continuous dynamical systems with discrete-time output measurements. The observability of this class of systems depends on the system inputs and on the output sampling time. In this context, we give sufficient conditions on both output sampling time and system input, which allow to preserve the observability of the system and consequently, guarantee the convergence of the continuous-discrete time observer synthesized in this paper.

In order to validate the theoretical results, the performance of the proposed observer is illustrated through an example dealing with biomass growth and bio-synthesis reactions.
\end{abstract}

keywords: Affine state systems, output injection, continuous-discrete time observer.

\title{
1. Introduction
}

By state affine systems up to output injection we mean systems of the form:

$$
\left\{\begin{array}{l}
\dot{x}=A(u, y) x+\varphi(u, y) \\
y=C x
\end{array}\right.
$$

The observer synthesis for these systems is widely investigated in the literature, see for instance [? ? ? ? ]. The proposed observers are obtained by using an approach similar to that of a Kalman filter [? ]. The main difficulty lies in the characterization of inputs for which the observer exponentially converges. Indeed, although system (1) is observable in the sense that it admits an input which renders it observable (universal input, see definition below), there may exist an input which makes it unobservable. Since the convergence of the observer is only guaranteed for inputs which make system (1) "asymptotically" observable, 
in [? ? ? ], the authors characterized this class of inputs and gave an exponential observer. This observer synthesis concerns continuous state affine systems with continuous time output measurement.

However, in many practical situations the output measurements are discrete-time ones. On the other hand, for single discrete output systems which are observable for any inputs, the authors in [?] showed that a continuous-discrete time extended Kalman filter converges exponentially if the sampling time is judiciously chosen. The proposed observer is based on a canonical form of uniformly observable systems ([? ]).

Contrary to the uniformly observable systems, the observability of system (1) depends on the inputs applied to the system (in the sense that it admits an input which makes it unobservable). Now, let us consider the continuous-discrete time state affine systems up to output injection of the form:

$$
\left\{\begin{array}{l}
\dot{x}=A(u, y) x+\varphi(u, y) \\
y\left(t_{k}\right)=C x\left(t_{k}\right),
\end{array}\right.
$$

Even if an input $u$ makes system (1) observable, there may exist an increasing sequence $\left(t_{k}\right)_{k \geq 0}$, with $\lim t_{k}=+\infty$ such that $u$ makes system (2) unobservable (see the counter example given in subsection 2.2).

This last remark implies that any observer construction necessarily depends on the input excitation and on the sampling time of the output measurements.

In this paper we give sufficient conditions on both output sampling time and system input, which allow to preserve the observability and permit to design an observer for state affine systems up to output injection with discrete output.

This paper is organized as follows: In section 2, we recall some observability properties 
of state affine systems and we give an observer synthesis for continuous-discrete times affine systems. In section 3, we extend this observer algorithm to continuous-discrete time state affine systems up to output injection. The considered systems are assumed to be disturbed. Finally, the performance of this observer is illustrated through a bioprocess model in order to validate the theoretical results and to demonstrate the usefulness of the proposed estimator in practical situations.

\section{Observer design for state affine systems}

\subsection{Continuous time observer}

Our goal here is to extend the well-known observer algorithm for continuous time state affine systems to continuous-discrete time affine systems. Therefore, we begin by recalling some properties concerning the observation of state affine systems. For more details, see [? ? ?]. State affine systems considered in the following are described by:

$$
\left\{\begin{array}{l}
\dot{x}=A(u) x+b(u) \\
y=C x
\end{array} x \in \mathbb{R}^{n}, u \in U \subset \mathbb{R}^{m}, y \in \mathbb{R}^{p},\right.
$$

where $x(t)$ is the unknown state, $u(t)$ is a known input, $y(t)$ is a known output, $A$ and $b$ depend continuously on $u$, and $C$ is a constant matrix.

Contrary to stationary linear systems, for nonlinear systems, the state estimation depends on the input excitations. We say that an input $u$ defined on some interval $\left[t_{0}, t_{0}+T\right]$ makes system (3) observable on $\left[t_{0}, t_{0}+T\right]$, or $\mathrm{u}$ is a universal input on $\left[t_{0}, t_{0}+T\right]$, if for every initial state $x_{0} \neq \bar{x}_{0}$; the associated outputs $y\left(x_{0}, u, t\right)$ and $y\left(\bar{x}_{0}, u, t\right)$ are not identically equal on $\left[t_{0}, t_{0}+T\right]$. 
Now, let $\phi_{u}\left(t, t_{0}\right)$ be the transition matrix of system (3):

$$
\left\{\begin{array}{l}
\frac{d}{d t} \phi_{u}\left(t, t_{0}\right)=A(u) \phi_{u}\left(t, t_{0}\right) \\
\phi\left(t_{0}, t_{0}\right)=I
\end{array}\right.
$$

where $\mathrm{I}$ is the $(n \times n)$ identity matrix.

For every $t_{1}, t_{2}, t_{3}$, the following equality holds:

$$
\phi_{u}\left(t_{1}, t_{2}\right) \phi_{u}\left(t_{2}, t_{3}\right)=\phi_{u}\left(t_{1}, t_{3}\right)
$$

In particular, $\phi_{u}(t, s)=\phi_{u}^{-1}(s, t)$.

Let us denote by:

$$
G\left(u, t_{0}, t_{0}+T\right)=\int_{t_{0}}^{t_{0}+T} \phi^{T}\left(t, t_{0}\right) C^{T} C \phi\left(t, t_{0}\right) d t
$$

the Grammian of observability of system (3). It is not difficult to see that $G\left(u, t_{0}, t_{0}+T\right)$ is a S.P.D. (symmetric positive definite) matrix if and only if $u$ is a universal input on $\left[t_{0}, t_{0}+T\right]$. In [? ? ], the authors designed an observer which exponentially converges for every input satisfying the following property:

Definition: A bounded input $u: \mathbb{R}^{+} \longrightarrow \mathbb{R}^{m}$ is called a regularly persistent input if:

$$
\exists T>0 ; \exists T_{0} \geq 0 ; \exists \alpha>0 ; \forall t \geq T_{0}, G(u, t, t+T) \geq \alpha I
$$

or equivalently, $\lambda_{\min }(G(u, t, t+T)) \geq \alpha$

In [? ? ], the authors stated some topological properties of the class of such inputs and 
showed that for every regularly persistent input, the following system:

$$
\left\{\begin{array}{l}
\dot{\hat{x}}=A(u) \hat{x}+b(u)-S^{-1} C^{T}(C \hat{x}-y) \\
\dot{S}=-\theta S-A^{T}(u) S-S A(u)+C^{T} C
\end{array}\right.
$$

is an exponential observer for system (3):

$\exists \theta_{0}>0 ; \forall \theta>\theta_{0} ; \forall S(0)$ a symmetric positive definite matrix; $\forall \hat{x}(0) \in \mathbb{R}^{n}$, we have

$$
\|\hat{x}(t)-x(t)\|^{2} \leq \mu e^{-\theta t}\|\hat{x}(0)-x(0)\|^{2}
$$

where $\mu$ is a positive constant.

\subsection{Continuous-discrete time observer}

As we have mentioned in the introduction, the time discretization of the output measurement may lead to the loss of observability. Indeed, consider the following bilinear system:

$$
\left\{\begin{array}{l}
\dot{x}=\left[\begin{array}{cc}
0 & u \\
-u & 0
\end{array}\right] x \\
y=x_{1}(t)
\end{array}\right.
$$

Noting that every admissible control $u:\left[t_{0}, t_{0}+T\right] \rightarrow \mathbb{R}$ which is not identically equal to zero, $u$ renders system (8) observable. In particular, (8) is observable for every constant input $u \neq 0$.

Now, let $u_{0}>0$ be any constant input, and let $\delta=\frac{\pi}{u_{0}}$ be any fixed sampling time constant, 
then $u_{0}$ makes the following continuous-discret time system:

$$
\left\{\begin{array}{l}
\dot{x}=\left[\begin{array}{cc}
0 & u \\
-u & 0
\end{array}\right] x \\
y(k . \delta)=x_{1}(k . \delta), \quad k=0,1,2, \ldots
\end{array}\right.
$$

unobservable.

Indeed, the outputs of (8) are:

$$
y(t)=x_{1}(0) \cos u_{0} t+\frac{x_{2}(0)}{u_{0}} \sin u_{0} t
$$

Now, let $x(0)=\left(\begin{array}{c}0 \\ x_{2}(0)\end{array}\right)$, with $x_{2}(0) \neq 0$, we obtain:

$$
y(k . \delta)=0, \forall k \geq 0
$$

Thus, the different initial conditions, $\left(\begin{array}{l}0 \\ 0\end{array}\right)$ and $\left(\begin{array}{c}0 \\ x_{2}(0)\end{array}\right)$ yield the same output trajectory. Hence, the input $u_{0}$ renders system (9) unobservable although it makes (8) observable.

Consequently, in this section, we will give a sufficient condition on the sampling time which permits to preserve the observability and to extend the observer stated in subsection 1 to continuous-discrete state affine systems.

Let us consider the continuous-discrete state affine system:

$$
\left\{\begin{array}{l}
\dot{x}=A(u) x+b(u) \\
y\left(t_{k}\right)=C x\left(t_{k}\right)
\end{array} x \in \mathbb{R}^{n}, u \in U \in \mathbb{R}^{m}, y\left(t_{k}\right) \in \mathbb{R}^{p}\right.
$$


where $\left(t_{k}\right)_{k \geq 0}$ is an increasing sequence such that $\lim _{k \rightarrow+\infty} t_{k}=+\infty$ and $\delta=\sup _{k \geq 0}\left(t_{k+1}-t_{k}\right)<+\infty$. Our candidate observer takes the form:

- For $t \in\left[t_{k}, t_{k+1}[\right.$

$$
\left\{\begin{array}{l}
\dot{\hat{x}}=A(u) \hat{x}+b(u) \\
\dot{S}=-\theta S-A^{T}(u) S-S A(u)
\end{array}\right.
$$

- For $t=t_{k+1}$

$$
\left\{\begin{array}{l}
S\left(t_{k+1}\right)=S^{-}\left(t_{k+1}\right)+\delta_{k} C^{T} C \\
\hat{x}\left(t_{k+1}\right)=\hat{x}^{-}\left(t_{k+1}\right)-\rho \delta_{k} S^{-1}\left(t_{k+1}\right) C^{T}\left(C \hat{x}^{-}\left(t_{k+1}\right)-y\left(t_{k+1}\right)\right)
\end{array}\right.
$$

where $S(0)$ is an arbitrary S.P.D. matrix, $\hat{x}(0)$ is also arbitrary and $\hat{x}^{-}\left(t_{k+1}\right)\left(\right.$ resp. $\left.S^{-}\left(t_{k+1}\right)\right)$ is the limit of $x(t)$ (resp. of $S(t))$ where $t \rightarrow t_{k+1}, t<t_{k+1}, \delta_{k}=t_{k+1}-t_{k}, \delta=\sup _{k \geq 0} \delta_{k}$ and $\rho \geq 1$ is a fixed parameter.

Theorem 2.1: Let u be a regularly persistent input for system (11), then system (12.13) is an exponential observer for system (11). i.e. :

$\exists \tilde{\theta}>0 ; \exists \tilde{\delta}>0 ; \forall \theta>\tilde{\theta} ; \forall \delta \in] 0, \tilde{\delta}] ; \forall S(0)$ a S.P.D. matrix; $\exists \mu_{1}>0 ; \exists \mu_{2}>0$ such that:

$$
\forall t \geq 0, \quad\|\hat{x}(t)-x(t)\|^{2} \leq \mu_{1} e^{-\mu_{2} t}\|\hat{x}(0)-x(0)\|^{2}
$$

where $x(t)$ is an unknown trajectory of (11) associated to the input $u ; \hat{x}(t)$ is any trajectory of system (12) associated to $(u, y)$

The proof of this theorem requires the following proposition: 
Proposition 2.1: Let $u$ be a regularly persistent input, then $\exists \tilde{\theta}>0 ; \exists \tilde{\delta}>0 ; \forall \theta \geq \tilde{\theta} ; \forall \delta \in$ ]0, $\tilde{\delta}] ; \forall S(0)$ a S.P.D. matrix; $\exists \beta_{1}>0 ; \exists \beta_{2}>0$ such that:

$$
\forall t \geq 0, \quad \beta_{1} I \leq S(t) \leq \beta_{2} I
$$

In the continuation, we proceed as follows: First we give the proof of theorem 2.1. Next, we prove proposition 2.1 .

\section{Proof: (Theorem 2.1)}

Setting $e(t)=\hat{x}(t)-x(t), e^{-}\left(t_{k}\right)=\lim _{\substack{t \rightarrow t_{k} \\ t<t_{k}}} e(t)$ and $S^{-}\left(t_{k}\right)=\lim _{\substack{t \rightarrow t_{k} \\ t<t_{k}}} S(t)$, we get:

$$
\left\{\begin{array}{l}
\dot{e}(t)=A(t) e(t) \text { for } t_{k} \leq t<t_{k+1} \\
e\left(t_{k+1}\right)=e^{-}\left(t_{k+1}\right)-\rho \delta_{k} S^{-1}\left(t_{k+1}\right) C^{T} C e^{-}\left(t_{k+1}\right)
\end{array}\right.
$$

Now, consider $V(t)=e(t)^{T} S(t) e(t)$, and $V^{-}\left(t_{k}\right)=\lim _{\substack{t \rightarrow t_{k} \\ t<t_{k}}} V(t)$

- For $t \in\left[t_{k}, t_{k+1}[\right.$, we have:

$$
\begin{aligned}
\dot{V}(t) & =2 e^{T} S \dot{e}+e^{T} \dot{S} e \\
& =2 e^{T} S \theta A(u) e-e^{T}\left(\theta S+A^{T}(u) S+S A(u)\right) e \\
& =-\theta V(t) .
\end{aligned}
$$

By simple integration we obtain:

$$
V(t)=e^{-\theta\left(t-t_{k}\right)} V\left(t_{k}\right)
$$

in particular, 


$$
V^{-}\left(t_{k+1}\right)=e^{-\theta \delta_{k}} V\left(t_{k}\right)
$$

- For $t=t_{k+1}$, the following equalities hold,

$$
\begin{aligned}
V\left(t_{k+1}\right) & =e^{T}\left(t_{k+1}\right) S\left(t_{k+1}\right) e\left(t_{k+1}\right) \\
& =\left(e^{-}\left(t_{k+1}\right)-\delta_{k} \rho S^{-1}\left(t_{k+1}\right) C^{T} C e^{-}\left(t_{k+1}\right)\right)^{T} S\left(t_{k+1}\right)\left(e^{-}\left(t_{k+1}\right)-\delta_{k} \rho S^{-1}\left(t_{k+1}\right) C^{T} C e^{-}\left(t_{k+1}\right)\right) \\
& =V^{-}\left(t_{k+1}\right)+\left(\delta_{k} \rho\right)^{2}\left(e^{-}\left(t_{k+1}\right)\right)^{T} C^{T} C S^{-1}\left(t_{k+1}\right) C^{T} C e^{-}\left(t_{k+1}\right)-\delta_{k}(2 \rho-1)\left\|C e^{-}\left(t_{k+1}\right)\right\|^{2} .
\end{aligned}
$$

From proposition 2.1, we obtain:

$$
V\left(t_{k+1}\right) \leq V^{-}\left(t_{k+1}\right)-\delta_{k}\left((2 \rho-1)-\frac{\delta_{k} \rho^{2}\|C\|^{2}}{\beta_{1}}\right)\left\|C e^{-}\left(t_{k+1}\right)\right\|^{2},
$$

Now we choose $\tilde{\delta}$ such that:

$$
\tilde{\delta} \leq \frac{(2 \rho-1) \beta_{1}}{\rho^{2}\|C\|^{2}}
$$

¿From (16) we get:

$$
V\left(t_{k+1}\right) \leq e^{-\theta \delta_{k}} V\left(t_{k}\right)
$$

Combining (15) and (19), we deduce that $\forall t \geq 0, \quad V(t) \leq e^{-\theta t} V(0)$.

Finally, using proposition 2.1, we get:

$$
\|e(t)\|^{2} \leq \mu_{1} e^{-\theta t}\|e(0)\|^{2}
$$

for some constant $\mu_{1}>0$, which depends on $\theta, S(0)$.

This ends the proof of theorem 2.1. 
Remark: If $u$ is a regularly persistent input, then a sufficient condition permitting to preserve the observability and to guaranty the convergence of the proposed observer (12 13) is given by (18).

The proof of proposition 2.1 requires some technical lemmas that we well give in the following. Let $a>0$ be a fixed real number and let us consider the functional space:

$E_{a}\left(\left[a_{1}, a_{2}\right], \mathbb{R}^{l}\right)=\left\{\varphi:\left[a_{1}, a_{2}\right] \rightarrow \mathbb{R}^{l}\right.$ which are differentiable on $\left[a_{1}, a_{2}\right]$ satisfying $\left.\sup _{t \in\left[a_{1}, a_{2}\right]}\left\|\frac{d \varphi}{d t}(t)\right\| \leq a\right\}$

Lemma 2.1: For every $\varepsilon>0$; for every sequence $\left(t_{k}\right)_{0 \leq k \leq N} ; a_{1}=t_{0}<t_{1}<\ldots<t_{N}=a_{2}$, such that $\sup _{0 \leq k \leq N-1}\left(t_{k+1}-t_{k}\right) \leq \frac{\varepsilon}{a \cdot\left(a_{2}-a_{1}\right)}$, we have:

$\forall \varphi \in E_{a}\left(\left[a_{1}, a_{2}\right], \mathbb{R}^{l}\right),\left\|\int_{a_{1}}^{a_{2}} \varphi(t) d t-\sum_{k=0}^{N-1}\left(t_{k+1}-t_{k}\right) \varphi\left(t_{k}\right)\right\| \leq \varepsilon$

Note that the sequence $\left(t_{k}\right)_{0 \leq k \leq N}$ does not depend on $\varphi$.

Proof: It suffices to give the proof for scalar functions.

Let $\varepsilon>0$ and let us consider any sequence $\left(t_{k}\right)_{0 \leq k \leq N}$ with $a_{1}=t_{0}<\ldots<t_{N}=a_{2}$ and such that $\sup _{0 \leq k \leq N-1}\left(t_{k+1}-t_{k}\right) \leq \frac{\varepsilon}{a \cdot\left(a_{1}-a_{2}\right)}$.

Let $\varphi \in E_{a}\left(\left[a_{1}, a_{2}\right], \mathbb{R}\right)$, we obtain:

$$
\left|\int_{a_{1}}^{a_{2}} \varphi(t) d t-\sum_{k=0}^{N-1}\left(t_{k+1}-t_{k}\right) \varphi\left(t_{k}\right)\right| \leq \sum_{k=0}^{N-1}\left|\int_{t_{k}}^{t_{k+1}} \varphi(t) d t-\left(t_{k+1}-t_{k}\right) \varphi\left(t_{k}\right)\right|
$$

Using the mean value theorem, we get: 


$$
\begin{aligned}
\int_{t_{k}}^{t_{k+1}} \varphi(t) d t=\left(t_{k+1}-t_{k}\right) \varphi\left(\tilde{t}_{k}\right), \text { for } \tilde{t}_{k} & \in\left[t_{k}, t_{k+1}\right], \text { hence: } \\
\left|\int_{a_{1}}^{a_{2}} \varphi(t) d t-\sum_{k=0}^{N-1}\left(t_{k+1}-t_{k}\right) \varphi\left(t_{k}\right)\right| & \leq \sum_{k=0}^{N-1}\left(t_{k+1}-t_{k}\right)\left|\varphi\left(\tilde{t}_{k}\right)-\varphi\left(t_{k}\right)\right| \\
& \leq a\left(a_{2}-a_{1}\right) \sup _{0 \leq k \leq N-1}\left(t_{k+1}-t_{k}\right)\left(\text { since } \varphi \in E_{a}\left(\left[a_{1}, a_{2}\right], \mathbb{R}\right)\right) \\
& \leq \varepsilon .
\end{aligned}
$$

Lemma 2.2: Let $u$ be a bounded input on $\mathbb{R}^{+}$and let $\tau>0$ be a constant, then:

$\forall t ; \forall s$, such that $|t-s|<\tau ; \forall M$ a S.P.D. matrix, we have:

$$
\lambda_{\min }(M) e^{-\lambda \tau} I \leq \phi_{u}^{T}(t, s) M \phi_{u}(t, s) \leq \lambda_{\max }(M) e^{\lambda \tau} I
$$

where $\lambda=2 \sup _{t \geq 0}\|A(u(t))\|, \lambda_{\min }(M),\left(\right.$ resp $\left.\lambda_{\max }(M)\right)$ stands for the smallest (resp. largest) eigenvalue of $M$ and $I$ is the identity matrix.

\section{Proof:}

- Proof of the second inequality of (20):

We know that: $\frac{d}{d t}\left[\phi_{u}^{T}(t, s) \phi_{u}(t, s)\right]=\phi_{u}^{T}(t, s)\left[A^{T}(u(t))+A(u(t))\right] \phi_{u}(t, s)$, therefore,

$$
\frac{d}{d t}\left[\phi_{u}^{T}(t, s) \phi_{u}(t, s)\right] \leq \lambda \phi_{u}^{T}(t, s) \phi_{u}(t, s)
$$

where $\lambda=2 \sup _{s \geq 0}\|A(u(s))\|$.

Hence, $\quad \phi_{u}^{T}(t, s) \phi_{u}(t, s) \leq e^{\lambda|t-s|} I$, 
then,

$$
\forall(t, s),|t-s| \leq \tau, \quad \phi_{u}(t, s) M \phi_{u}^{T}(t, s) \leq \lambda_{\max }(M) e^{\lambda|t-s|} I
$$

- Proof of the first inequality of (20):

Using a similar argument as in (22), we obtain:

$$
\begin{aligned}
\phi_{u}(s, t) M^{-1} \phi_{u}^{T}(s, t) & \leq \lambda_{\max }\left(M^{-1}\right) e^{\lambda|t-s|} I \\
& =\frac{e^{\lambda|t-s|}}{\lambda_{\min }(M)} I .
\end{aligned}
$$

Hence,

$$
\begin{aligned}
\phi_{u}^{T}(t, s) M \phi_{u}(t, s) & =\left[\phi_{u}(s, t) M^{-1} \phi_{u}^{T}(s, t)\right]^{-1} \\
& \geq \lambda_{\min }(M) e^{-\lambda|t-s|} I .
\end{aligned}
$$

Now, let us give the proof of proposition 2.1

\section{Proof: Proposition 2.1}

From (12), (13), we obtain:

For $t \in\left[t_{k}, t_{k+1}[\right.$,

$$
S(t)=e^{-\theta\left(t-t_{k}\right)} \phi_{u}^{T}\left(t_{k}, t\right) S\left(t_{k}\right) \phi_{u}\left(t_{k}, t\right)
$$

For $t=t_{k+1}$,

$$
S\left(t_{k+1}\right)=e^{-\theta \delta_{k}} \phi_{u}^{T}\left(t_{k}, t_{k+1}\right) S\left(t_{k}\right) \phi_{u}\left(t_{k}, t_{k+1}\right)+\delta_{k} C^{T} C,
$$

where $\delta_{k}=t_{k+1}-t_{k}$ and $\phi_{u}^{T}\left(t_{k}, t\right)=\left(\phi_{u}^{T}\left(t, t_{k}\right)\right)^{-1}$ 
Let $\delta=\sup _{k \geq 0} \delta_{k}<+\infty$.

Combining (25-26) and using lemma 2.2, the proof of inequalities (14) of proposition 2.1 can be reduced to the proof of the following:

$\exists \tilde{\delta}>0 ; \exists \tilde{\theta}>0 ; \forall \theta \geq \tilde{\theta} ; \forall \delta \in] 0, \tilde{\delta}] ; \exists \beta_{1}, \beta_{2}>0$ such that,

$$
\forall k \geq 0, \quad \beta_{1} I \leq S\left(t_{k}\right) \leq \beta_{2} I
$$

- Proof of the second inequality of (27):

From (25),(26), we deduce that:

$$
\begin{aligned}
S\left(t_{k+1}\right) & =e^{-\theta t_{k+1}} \phi^{T}\left(0, t_{k+1}\right) S(0) \phi_{u}\left(0, t_{k+1}\right) \\
& +\sum_{i=0}^{k} \delta_{i} e^{-\theta\left(t_{k+1}-t_{i+1}\right)} \phi^{T}\left(t_{i+1}, t_{k+1}\right) C^{T} C \phi\left(t_{i+1}, t_{k+1}\right)
\end{aligned}
$$

Since $u$ is a bounded input, from lemma 2.2, we have:

$\phi_{u}^{T}\left(t_{i}, t_{k}\right) M \phi_{u}\left(t_{i}, t_{k}\right) \leq\|M\| e^{\lambda\left(t_{k}-t_{i}\right)} I$, for $0 \leq i \leq k$, where $\|M\|$ denotes the $L_{2}-$ norm of $M$. Thus,

$$
S\left(t_{k+1}\right) \leq\left(e^{-(\theta-\lambda) t_{k+1}}\|S(0)\|+\sum_{i=0}^{k} \delta_{i} e^{-(\theta-\lambda)\left(t_{k+1}-t_{i+1}\right)}\left\|C^{T} C\right\|\right) I .
$$

Now, taking for $\tilde{\theta}$ any constant such that $\tilde{\theta}>\lambda$, and $\theta \geq \tilde{\theta}$; there exists a constant $\beta_{2}>0$ such that:

$$
\forall k \geq 0, \quad S\left(t_{k}\right) \leq \beta_{2} I
$$

- Proof of the first inequality of (27): 
Let $u$ be a regularly persistent input, which means that $u$ is a bounded input satisfying:

$$
\int_{t}^{t+T} \phi_{u}^{T}(s, t) C^{T} C \phi_{u}(s, t) d s \geq \alpha I,
$$

for every $t \geq T_{0}$, where $T_{0}, T$, and $\alpha>0$ are constants.

Now, for every $t \geq 0$, denote by $u_{t}$ the mapping:

$$
\begin{aligned}
u_{t}:[0, T] & \longrightarrow \mathbb{R} \\
s & \longmapsto u_{t}(s)=u(t+s) .
\end{aligned}
$$

¿From the definition of $\phi_{u}$, it is easy to verify that:

$$
\forall t \geq 0, \forall s \in[0, T] \quad \phi_{u_{t}}(s, 0)=\phi_{u}(s+t, t) .
$$

Hence, (30) is equivalent to:

$$
\begin{aligned}
\forall t \geq T_{0}, \quad \int_{t}^{t+T} \phi_{u}^{T}(s, t) C^{T} C \phi_{u}(s, t) d s & =\int_{0}^{T} \phi_{u_{t}}^{T}(s, 0) C^{T} C \phi_{u_{t}}(s, 0) d s \\
& \geq \alpha I
\end{aligned}
$$

Set $k_{0}=\inf \left\{l / t_{l} \geq T_{0}+T\right\}$ and for every $k \geq k_{0}$, set $\nu(k)=\sup \left\{l / t_{l+1} \leq t_{k+1}, t_{k+1}-t_{l+1} \geq\right.$ $T\}$ and finally, set $\delta=\sup _{k \geq 0} \delta_{k}$. We obtain:

$$
T \leq t_{k+1}-t_{\nu(k)} \leq T+\delta
$$

To prove the first inequality of (27), we examine the following two cases: 
Case 1: $k \geq k_{0}$

Expression (28) leads to:

$$
\begin{aligned}
S\left(t_{k+1}\right) & \geq \sum_{i=\nu(k)}^{k} \delta_{i} e^{-\theta\left(t_{k+1}-t_{i+1}\right)} \phi_{u}^{T}\left(t_{i+1}, t_{k+1}\right) C^{T} C \phi_{u}\left(t_{i+1}, t_{k+1}\right) \\
& \geq \sum_{i=0}^{k-\nu(k)} \tilde{\delta}_{i} e^{-\theta\left(\tilde{t}_{k+1-\nu(k)}-\tilde{t}_{i+1}\right)} \phi_{u_{\nu(k)}}^{T}\left(\tilde{t}_{i+1}, \tilde{t}_{k+1-\nu(k)}\right) C^{T} C \phi_{u_{\nu}(k)}\left(\tilde{t}_{i+1}, \tilde{t}_{k+1-\nu(k)}\right)
\end{aligned}
$$

where $\tilde{t}_{i}=t_{i+\nu(k)}-t_{\nu(k)}$ and $\tilde{\delta}_{i}=\delta_{i+\nu(k)}$.

Thus,

$$
\begin{aligned}
S\left(t_{k+1}\right) & \geq e^{-\theta T} \sum_{i=0}^{k-\nu(k)} \tilde{\delta}_{i} \phi_{u_{t_{\nu}(k)}^{T}}^{T}\left(\tilde{t}_{i+1}, \tilde{t}_{k+1-\nu(k)}\right) C^{T} C \phi_{u_{t_{\nu}(k)}}\left(\tilde{t}_{i+1}, \tilde{t}_{k+1-\nu(k)}\right) \\
& =e^{-\theta T} \phi_{u_{t_{\nu}(k)}}^{T}\left(0, \tilde{t}_{k+1-\nu(k)}\right)\left[\sum_{i=0}^{k-\nu(k)} \tilde{\delta}_{i} \phi_{u_{\nu(k)}}^{T}\left(\tilde{t}_{i+1}, 0\right) C^{T} C \phi_{u_{t_{\nu}(k)}}\left(\tilde{t}_{i+1}, 0\right)\right] \phi_{u_{t_{\nu(k)}}}\left(0, \tilde{t}_{k+1-\nu(k)}\right)
\end{aligned}
$$

From (32), we know that $T \leq \tilde{t}_{k+1-\nu(k)} \leq T+\delta$, where $\delta=\sup _{k \geq 0} \delta_{k}$.

Now combining this last fact with (33) and using lemma 2.2, it follows:

$$
\begin{aligned}
S\left(t_{k+1}\right) & \geq e^{-\theta T} e^{-\lambda \tilde{t}_{k+1-\nu(k)}} \lambda_{\min }\left(\sum_{i=0}^{k-\nu(k)} \tilde{\delta}_{i} \phi_{u_{\nu(k)}}^{T}\left(\tilde{t}_{i+1}, 0\right) C^{T} C \phi_{u_{t_{\nu}(k)}}\left(\tilde{t}_{i+1}, 0\right)\right) \\
& \geq e^{-\theta T} e^{-\lambda(T+\delta)} \lambda_{\min }\left(\sum_{i=0}^{k-\nu(k)} \tilde{\delta}_{i} \phi_{u_{t_{\nu(k)}}}^{T}\left(\tilde{t}_{i+1}, 0\right) C^{T} C \phi_{u_{t_{\nu}(k)}}\left(\tilde{t}_{i+1}, 0\right)\right)
\end{aligned}
$$

where $\lambda_{\min }$ stands for the smallest eigenvalue.

Consequently, to show that $S\left(t_{k+1}\right) \geq \beta I, \quad \forall k \geq k_{0}$, for some constant $\beta>0$, it suffices to show that:

$$
\inf _{k \geq k_{0}}\left(\lambda_{\min }\left(\sum_{i=0}^{k-\nu(k)} \tilde{\delta}_{i} \phi_{u_{t_{\nu(k)}}^{T}}^{T}\left(\tilde{t}_{i+1}, 0\right) C^{T} C \phi_{u_{t_{\nu(k)}}}\left(\tilde{t}_{i+1}, 0\right)\right)\right)>0
$$


To prove (35), let us remark that for every $k \geq k_{0}$, the restriction of $\phi^{T} u_{t_{\nu(k)}}(s, 0) C^{T} C \phi_{u_{t_{\nu}(k)}}(s, 0)$ to $\left[0, \tilde{t}_{k+1}\right]$ belongs to $E_{a}\left(\left[0, \tilde{t}_{k+1}\right], \mathbb{R}^{n^{2}}\right)($ defined in lemma 2.1$)$, where $a=\lambda\left\|C^{T} C\right\| e^{\lambda(T+\delta)}$.

Indeed,

$$
\begin{aligned}
\left\|\frac{d}{d s} \phi_{u_{t_{\nu}(k)}}^{T}(s, 0) C^{T} C \phi_{u_{t_{\nu(k)}}}(s, 0)\right\|= & \| \phi_{u_{t_{\nu(k)}}}^{T}(s, 0)\left[A^{T}\left(u_{t_{\nu(k)}}(s)\right) C^{T} C C^{T} C A\left(u_{t_{\nu(k)}}(s)\right)\right] \\
& \phi_{u_{t_{\nu(k)}}}(s, 0) \|,
\end{aligned}
$$

and by lemma 2.2 we get:

$$
\begin{aligned}
\sup _{s \in\left[0, t_{k}\right]}\left\|\frac{d}{d s} \phi^{T}{ }_{u_{t_{\nu(k)}}}(s, 0) C^{T} C \phi_{u_{t_{\nu}(k)}}(s, 0)\right\| & \leq \lambda\left\|C^{T} C\right\| e^{\lambda \tilde{t}_{k+1}} \\
& \leq \lambda\left\|C^{T} C\right\| e^{\lambda(T+\delta)} \quad\left(\text { since } \quad \tilde{t}_{k+1} \leq T+\delta\right) .
\end{aligned}
$$

Now, applying lemma 2.1 with $\varepsilon=\frac{\alpha}{2}$, and $\alpha$ being the constant given in (31), we get:

$\left\|\int_{0}^{\tilde{t}_{k+1}} \phi_{u_{t_{\nu}(k)}}^{T}(s, 0) C^{T} C \phi_{u_{t_{k}}}(s, 0) d s-\sum_{i=0}^{N-1}\left(\tau_{i+1}-\tau_{i}\right) \phi^{T}{ }_{u_{t_{\nu}(k)}}\left(\tau_{i}, 0\right) C^{T} C \phi_{u_{t_{\nu(k)}}}\left(\tau_{i}, 0\right)\right\| \leq \frac{\alpha}{2}$,

for every sequence $\left(\tau_{i}\right)_{0 \leq i \leq N}, \quad 0=\tau_{0}<\tau_{1}<\ldots<\tau_{N}=\tilde{t}_{k+1}$ such that $\sup _{0 \leq k \leq N}\left(\tau_{i+1}-\tau_{i}\right) \leq \frac{\varepsilon}{a \cdot \tilde{t}_{k+1}}=\frac{\alpha e^{-\lambda(T+\delta)}}{2 \lambda\left\|C^{T} C\right\| \tilde{t}_{k+1}}$.

In particular, this is true for $\tau_{i}=\tilde{t}_{i+1}$ with $\sup _{\nu(k) \leq i \leq k}\left(t_{i+1}-t_{i}\right) \leq \frac{\alpha e^{-\lambda(T+\delta)}}{2 \lambda\left\|C^{T} C\right\|(T+\delta)}$. Thus for $\delta \leq \frac{\alpha e^{-\lambda(T+\delta)}}{2 \lambda\left\|C^{T} C\right\|(T+\delta)}$ and $k \geq k_{0},(35)$ is satisfied.

Case 2: $k \leq k_{0}$ :

Clearly, $\forall k, S\left(t_{k}\right)$ is a S.P.D. matrix, hence $S\left(t_{k}\right) \geq \beta(k) I$, where $\beta(k)>0$ is a constant. 
Now, taking $\beta_{k_{0}}=\inf _{0 \leq k \leq k_{0}+1} \beta(k)$ it follows:

$$
S\left(t_{k}\right) \geq \beta_{k_{0}} I
$$

Finally, choosing $\beta_{1}$ to be the smallest value of the two constants obtained from case 1 and case 2, we have:

$$
\forall k \geq 0, \quad S\left(t_{k}\right) \geq \beta_{1} I
$$

This ends the proof of proposition 2.1

In the following section, we will give a generalization of the last result in the sense that the state coefficients can depend on the output and the model takes into account the possible presence of a perturbation.

\section{State estimation for disturbed state affine systems up to output injection}

The aim here consists in extending the continuous-discrete time estimator given above, to the class of nonlinear continuous-discrete disturbed state affine systems up to output injection taking the form:

$$
\left\{\begin{array}{l}
\dot{x}=A(u, y) x+b(u, y)+d(t, x) \\
y\left(t_{k}\right)=C x\left(t_{k}\right) \quad x \in \mathbb{R}^{n}, u \in \mathbb{R}^{m}, y \in \mathbb{R}^{p},
\end{array}\right.
$$

where $d(t, x)$ is a bounded and unknown function.

Earlier, in [?], the authors gave an exponential observer for the following continuous-time 
state affine systems up to output injection:

$$
\left\{\begin{array}{l}
\dot{x}=A(u, y) x+b(u, y) \\
y=C x
\end{array}\right.
$$

And they shown that if $(u, y)$ is a regularly persistent input for the system:

$$
\left\{\begin{array}{l}
\dot{\xi}=A(u, y) \xi \\
z=C . \xi
\end{array}\right.
$$

then, system (40) forms an exponential for (38) (see [?]).

$$
\left\{\begin{array}{l}
\dot{\hat{x}}=A(u, y) \hat{x}+b(u, y)-\rho S^{-1} C^{T}(C \hat{x}-y) \\
\dot{S}=-\theta S-A^{T}(u, y) S-S A(u, y)+C^{T} C
\end{array}\right.
$$

This observer design is based on the following claim:

Claim 1: $u$ is universal input on $[0, T]$ for (38) if and only if $(u, y)$ is a universal input on $[0, T]$ for system (39).

Now, instead of continuous-time measurements, we consider here the state estimation based on discrete-time observation $y\left(t_{k}\right)=C x\left(t_{k}\right)$, where $\left(t_{k}\right)_{k \geq 0}$ is an increasing sequence, with $\lim _{k \rightarrow+\infty} t_{k}=+\infty$ and $\sup _{k \geq 0}\left(t_{k+1}-t_{k}\right)<+\infty$.

Our proposed estimator takes the form: 
- For $t \in\left[t_{k}, t_{k+1}[\right.$

$$
\left\{\begin{array}{l}
\dot{\hat{x}}(t)=A\left(u(t), y\left(t_{k}\right)\right) \hat{x}(t)+b\left(u(t), y\left(t_{k}\right)\right) \\
\dot{S}(t)=-\theta S(t)-A^{T}\left(u(t), y\left(t_{k}\right)\right) S(t)-S(t) A\left(u(t),\left(t_{k}\right)\right)
\end{array}\right.
$$

- For $t=t_{k+1}$

$$
\left\{\begin{array}{l}
\hat{x}\left(t_{k+1}\right)=\hat{x}^{-}\left(t_{k+1}\right)-\rho \delta_{k} S^{-1}\left(t_{k+1}\right) C^{T}\left(C \hat{x}^{-}\left(t_{k+1}\right)-y\left(t_{k+1}\right)\right) \\
S\left(t_{k+1}\right)=S^{-}\left(t_{k+1}\right)+\delta_{k} C^{T} C
\end{array}\right.
$$

where $S(0)$ is a S.P.D matrix, $\delta_{k}=t_{k+1}-t_{k}$ and $\rho$ is a constant such that $\rho \geq 1$.

The convergence of the above estimator is based on the following assumptions:

(H1) There exists a class of bounded inputs denoted by $u \in \mathcal{U} \subset L^{\infty}\left(\mathbb{R}^{+}, U\right)$ and two bounded sets $K_{1} \subset K_{2}$ of $\mathbb{R}^{n}$ such that for every $\mathcal{U}$ and every initial state $x_{0} \in K_{1}$, the trajectory $x(t)$ of $(\underline{38})$ associated to $x_{0}$ and $u$ lies in $K_{2}$.

(H2) For every $u \in \mathcal{U}$ and every initial state $x_{0} \in K_{1}$, the associated output $y$ is such that $(u, y)$ becomes a regularly persistent input of the system (39).

Now, we can state the main result of this section.

Theorem 3.1: Under hypothesis (H1) and (H2), we have:

$\exists \tilde{\theta}>0 ; \exists \tilde{\delta}>0 ; \forall \delta \in] 0, \tilde{\delta}] ; \forall \theta \geq \tilde{\theta} ; \forall S(0)$ a S.P.D. matrix; $\exists \gamma_{1}>0 ; \exists \gamma_{2}>0 ; \exists \gamma_{3}>0$ such that:

$$
\forall t \geq 0, \quad\|\hat{x}(t)-x(t)\| \leq \gamma_{1} e^{-\gamma_{2} t}\|\hat{x}(0)-x(0)\|+\gamma_{3}
$$

where

$$
\delta=\sup _{k \geq 0} \delta_{k}, \quad \delta_{k}=t_{k+1}-t_{k}, x(0) \in K_{1} \text { and } \hat{x}(0) \in \mathbb{R}^{n} .
$$


$\gamma_{3}$ becomes small as soon as $\sup _{k \geq 0, t \in\left[t_{k}, t_{k+1}\right]}\left\|y(t)-y\left(t_{k}\right)\right\|$ and $\sup _{(x, t)}\|d(t, x)\|$ becomes small.

In the sequel, we will denote by $\tilde{y}$ the function defined by $\tilde{y}(t)=y\left(t_{k}\right)$ for $t_{k} \leq t<t_{k+1}$, $k=0,1 \ldots$

The proof of theorem 3.1 requires the following lemma:

Lemma 3.1: Under hypothesis (H1) and (H2), there exists a $\delta^{\sharp}>0$ such that for every $\left.\delta \in] 0, \delta^{\sharp}\right],(u, \tilde{y})$ becomes a regularly persistent input for system (39).

\section{Proof:}

Let $u \in \mathcal{U}$ and $x(0) \in K_{1}$, from $(\mathrm{H} 2)$, we know that $(u, y)$ is a regularly persistent input:

$$
\exists T_{0} \geq 0 ; \exists \alpha>0 ; \exists T>0 ; \forall t \geq T_{0}, \quad \int_{t}^{t+T} \psi_{w}^{T}(s, t) C^{T} C \psi_{w}(s, t) d s \geq \alpha I
$$

where,

$$
\left\{\begin{array}{l}
\frac{d}{d s} \psi_{w}(s, t)=A(w(s)) \psi_{w}(s, t) \\
\psi_{w}(t, t)=0
\end{array}\right.
$$

Using the notations of section 2 (see(31)), the expression (43) can be rewritten as:

$$
\int_{0}^{T} \psi_{w_{t}}^{T}(s, 0) C^{T} C \psi_{w_{t}}(s, 0) d s \geq \alpha I, \quad \forall t \geq t_{0}
$$

Let $L^{\infty}\left([0, T], \mathbb{R}^{N}\right)$ denotes the space of the bounded borelian functions from $[0, T]$ into $\mathbb{R}^{N}$, which is induced with the norm $\|v\|_{\infty}=\sup _{t \in[0, T]}\|v(t)\|$. It is not difficult to see that the 
Grammian map:

$$
\begin{aligned}
L^{\infty}\left([0, T], \mathbb{R}^{m+p}\right) & \longrightarrow \mathbb{R}^{n^{2}} \\
w(\cdot) & \longmapsto \int_{0}^{T} \psi_{w}^{T}(\cdot, 0) C^{T} C \psi_{w}(\cdot, 0),
\end{aligned}
$$

is a continuous map.

Now, using the mean value theorem and the fact that $x(t) \in K_{2}, \forall t \geq 0$ (hypotheses (H1)), we get:

$$
\begin{aligned}
\left\|y_{t}(\cdot)-\tilde{y}_{t}(\cdot)\right\|_{L^{\infty}\left([0, L], \mathbb{R}^{P}\right)} & \leq \delta\|\dot{y}(\cdot)\|_{L^{\infty}\left([0, L], \mathbb{R}^{P}\right)}, \text { where }\left(\delta=\sup _{k \geq 0} \delta_{k}\right) \\
& =\delta\|C A(u(\cdot), y(\cdot)) x(\cdot)\|_{L^{\infty}\left([0, L], \mathbb{R}^{P}\right)} \\
& \leq \gamma . \delta
\end{aligned}
$$

where $\gamma$ is a constant which depends on the bounded set $K_{2}$ and $u$.

Now, combining this last inequality with (44) and the continuity of the above Grammian map, we can find constants $\delta^{\sharp}>0$ and $\alpha^{\sharp}>0$, such that for every $\left.\left.\delta \in\right] 0, \delta^{\sharp}\right]$ and every $t \geq T_{0}$, we have:

$$
\int_{0}^{T} \psi_{\left(u_{t}, \tilde{y}_{t}\right)}^{T}(s, 0) C^{T} C \phi_{\left(u_{t}, \tilde{y}_{t}\right)}(s, 0) d s \geq \alpha^{\sharp} I .
$$

Hence $(u, \tilde{y})$ is a regularly persistent input for system (39)

\section{Proof: Theorem 3.1}

Set $e(t)=\hat{x}(t)-x(t)$ and,

$\Delta A=A(u, \tilde{y})-A(u, y), \quad \Delta b=b(u, \tilde{y})-b(u, y)$

we get: 
- For $t \in\left[t_{k}, t_{k+1}[\right.$,

$$
\left\{\begin{array}{l}
\dot{e}(t)=A(u(t), \tilde{y}(t)) e(t)+\Delta A x(t)+\Delta b-d(t) \\
\dot{S}(t)=-\theta S(t)-A^{T}(u(t), \tilde{y}(t)) S(t)-S(t) A(u(t), \tilde{y}(t))
\end{array}\right.
$$

- For $t=t_{k+1}$,

$$
\left\{\begin{array}{l}
e\left(t_{k+1}\right)=e^{-}\left(t_{k+1}\right)-\rho \delta_{k} S^{-1}\left(t_{k+1}\right) C^{T} C e^{-}\left(t_{k+1}\right) \\
S\left(t_{k+1}\right)=S^{-}\left(t_{k+1}\right)+\delta_{k} C^{T} C
\end{array}\right.
$$

Now, considering the quadratic function $V(t)=e(t)^{T} S(t) e(t)$, we obtain:

- For $t \in\left[t_{k}, t_{k+1}[\right.$,

$$
\begin{aligned}
\dot{V} & =-\theta V+2 e^{T}(t) S(t)[\Delta A(t) x(t)+\Delta b(t)-d(t)] \\
& \leq-\theta V(t)+2 \sqrt{V(t)}[(\Delta A(t) x(t)+\Delta b(t)-d(t)) S(t)(\Delta A(t) x(t)+\Delta b(t)-d(t))]^{1 / 2}
\end{aligned}
$$

¿From lemma 3.1 , we know that $(u, \tilde{y})$ is a regularly persistent input.

Applying proposition 2.1 we deduce that there exist two constants $\beta_{1}>0$ and $\beta_{2}>0$ only depending on $\delta^{\sharp}, \theta$ and $(u, y)$ such that for every $\left.\left.\delta \in\right] 0, \delta^{\sharp}\right]$ we have:

$$
\forall t \geq 0, \quad \beta_{1} I \leq S(t) \leq \beta_{2} I
$$

Combining (47) and (48),we deduce that:

$$
\dot{V}(t) \leq-\theta V(t)+2 \sqrt{\beta_{2}} \sqrt{V(t)}\|\Delta A x(t)+\Delta b-d(t)\|^{2},
$$


or equivalently,

$$
\frac{d}{d t}(\sqrt{V(t)}) \leq-\frac{\theta}{2} \sqrt{V(t)}+\sqrt{\beta_{2}}\|\Delta A x(t)+\Delta b(t)-d(t)\| .
$$

Thus,

$$
\text { for } t_{k} \leq t<t_{k+1} \quad \sqrt{V(t)} \leq e^{-\frac{\theta}{2}\left(t-t_{k}\right)} \sqrt{V\left(t_{k}\right)}+\frac{2 \mu}{\theta} \sqrt{\beta_{2}} \text {. }
$$

where,

$$
\mu=\sup _{t \geq 0}\|\Delta A x(t)+\Delta b-d(t)\|
$$

- For $t=t_{k+1}$,

As in the proof of theorem 2.1 (see (17)), the following inequality holds :

$$
V\left(t_{k+1}\right) \leq V^{-}\left(t_{k+1}\right)-\left(\delta_{k}(2 \rho-1)-\frac{\delta_{k}^{2} \rho^{2}\|C\|^{2}}{\beta_{1}}\right)\left\|C e^{-}\left(t_{k+1}\right)\right\|^{2}
$$

Thus, for $\delta$ such that $\delta(2 \rho-1)-\frac{\delta^{2} \rho^{2}\|C\|^{2}}{\beta_{1}} \geq 0$, (i.e. $\delta \leq \frac{(2 \rho-1) \beta_{1}}{\rho^{2}\|C\|^{2}}$, we obtain:

$$
\begin{aligned}
\sqrt{V\left(t_{k+1}\right)} & \leq \sqrt{V^{-}\left(t_{k+1}\right)} \\
& =\sqrt{e^{-}\left(t_{k+1}\right) S^{-}\left(t_{k+1}\right) e^{-}\left(t_{k+1}\right)} \\
& \leq e^{-\theta \delta_{k}} \sqrt{V\left(t_{k}\right)}+\frac{2 \mu \sqrt{\beta_{2}}}{\theta}
\end{aligned}
$$

Thus for $\left.\delta \in] 0, \min \left(\frac{(2 \rho-1) \beta_{1}}{\left(\rho^{2}\|C\|^{2}\right.}, \delta^{\sharp}\right)\right]$, the inequality (49) together with (51) implies that $e(t)$ 
is attracted by a neighbourhood of the origin which depends on the constant $\frac{2 \mu \sqrt{\beta_{2}}}{\theta}$, where $\mu$ is given by $(50)$.

Noticing that this neighbourhood is small if $\mu$ is small. This is the case when $\|\tilde{y}(t)-y(t)\|_{L^{\infty}}$ and the perturbation norm $\|d(t)\|_{L^{\infty}}$ are small.

\section{Application to a biological denitrification process}

In this section we present an the application of the continuous-discrete observer developed in the section above to the estimation of kinetic reactions in biological denitrification.

\subsection{Model representation}

This process is defined as the dissimilatory reduction, by anaerobic bacteria (Pseudomonas denitrificans (ATCC 13867)), of one or both of the ionic nitrogen oxides: nitrate and nitrite, to gaseous nitrogen products by using organic substrates. The bio-degradation takes place through two principal steps:

$$
\mathrm{NO}_{3}^{-} \longrightarrow \mathrm{NO}_{2}^{-} \longrightarrow \mathrm{N}_{2}
$$

The dynamic model that we use takes into account the accumulation of nitrite and the inhibition by nitrate [?]. It is built based on the kinetics of substrates consumption and it is given by the following system:

$$
\left\{\begin{array}{l}
\dot{x}=\left(\mu_{1}+\mu_{2}\right) x-D x \\
\dot{s_{1}}=-y_{11} \mu_{1} x-D\left(s_{1}-s_{1, i n}\right) \\
\dot{s_{2}}=\left(y_{12} \mu_{1}-y_{22} \mu_{2}\right) x-D s_{2} \\
\dot{s_{3}}=-\left(y_{13} \mu_{1}+y_{23} \mu_{2}\right) x-D\left(s_{3}-s_{3, \text { in }}\right)
\end{array}\right.
$$


where $x, s_{1}, s_{2}$ and $s_{3}$, represent respectively, the cell mass, the nitrate, nitrite and acetic acid concentrations. $D$ is the dilution rate and $s_{i, i n}(i=1,3)$ is the concentration of the substrate in the inlet stream. $y_{i j}$ are yield coefficients and are assumed to be constants.

In previous work [?], it is shown that under some operator conditions the specific growth rates $\mu_{i}(i=1,2)$ can be modeled using the well known Monod law:

$$
\left\{\begin{array}{l}
\mu_{1}=\mu_{1 m} \frac{s_{1}}{s_{1}+k_{1}} \frac{s_{3}}{s_{3}+k_{3}} \\
\mu_{2}=\mu_{2 m} \frac{s_{2}}{s_{2}+k_{2}} \frac{k_{I}}{s_{1}+k_{I}} \frac{s_{3}}{s_{3}+k_{3}}
\end{array}\right.
$$

where $\mu_{i m}(i=1,2)$ are the maximal specific growth rates for the cells grown, $k_{i}(i=1,2,3)$ are, respectively, the nitrate and nitrite half saturation constants and $k_{I}$ is the nitrate inhibition constant. However, it is well-known that for complex bioprocesses, where many components and bioreactions take place, $\mu_{i}(t)$ can have a more complex representation and identification results are sensitive to operator conditions. This explains the interest in representing $\mu_{i}(t)$ as a state variable which needs to be estimated.

\subsection{Observer for the bioreactor model}

Let us use the following notations:

$$
\mu_{1}=\alpha_{1} s_{3} \quad \text { and } \quad \mu_{2}=\alpha_{2} s_{3}
$$

In the following, we show how we can estimate $\alpha_{i}(t)$ (consequently $\mu_{i}$ ) using biomass and organic substrate concentrations as the only available discrete time measurements. To do so, we will introduce the following reduced model: 


$$
\left\{\begin{array}{l}
\dot{x}=\left(\alpha_{1}+\alpha_{2}\right) x s_{3}-D x \\
\dot{s_{3}}=-\left(y_{13} \alpha_{1}+y_{23} \alpha_{2}\right) x s_{3}-D\left(s_{3}-s_{3, i n}\right) \\
y\left(t_{k}\right)=\left(\begin{array}{c}
x \\
s_{3}
\end{array}\right)
\end{array}\right.
$$

It is well known that the parameter $\alpha_{i}(t)$ is not constant, it must be treated as a time varying parameter which can depend on biomass, substrates and operator conditions (see [? ? ? ]). Moreover, its dynamics is not well known since it is subject to many causes of disturbances and even theoretical uncertainties. Consequently, system (54) can be extended as follows:

$$
\left\{\begin{array}{l}
\dot{x}=\left(\alpha_{1}+\alpha_{2}\right) x s_{3}-D x \\
\dot{s_{3}}=-\left(y_{13} \alpha_{1}+y_{23} \alpha_{2}\right) x s_{3}-D\left(s_{3}-s_{3, i n}\right) \\
\dot{\alpha}_{1}=\tilde{\alpha}_{1} \\
\dot{\alpha}_{2}=\tilde{\alpha}_{2} \\
\dot{\tilde{\alpha}}_{1}=\varepsilon_{1}(t) \\
\dot{\tilde{\alpha}}_{2}=\varepsilon_{2}(t) \\
y\left(\begin{array}{c}
x \\
\left.s_{k}\right)
\end{array}\right)
\end{array}\right.
$$

where $\varepsilon_{i}(t)$ are unknown and bounded functions which may depend on the states, the inputs, noise, etc.

System (55) can be rewritten as follows:

$$
\begin{aligned}
\dot{X}(t) & =A(y(t), u(t)) X(t)+b(y(t), u(t))+d(t) \\
y\left(t_{k}\right) & =C X\left(t_{k}\right)
\end{aligned}
$$


where

$$
\begin{aligned}
& X(t)=\left[\begin{array}{c}
x \\
s_{3} \\
\alpha_{1} \\
\alpha_{2} \\
\tilde{\alpha_{1}} \\
\tilde{\alpha_{2}}
\end{array}\right] A=\left[\begin{array}{ccccccc}
0 & 0 & x s_{3} & x s_{3} & 0 & 0 \\
0 & 0 & -y_{13} x s_{3} & -y_{23} x s_{3} & 0 & 0 \\
0 & 0 & 0 & 0 & 1 & 1 \\
0 & 0 & 0 & 0 & 1 & 1 \\
0 & 0 & 0 & 0 & 0 & 0 \\
0 & 0 & 0 & 0 & 0 & 0
\end{array}\right]
\end{aligned}
$$

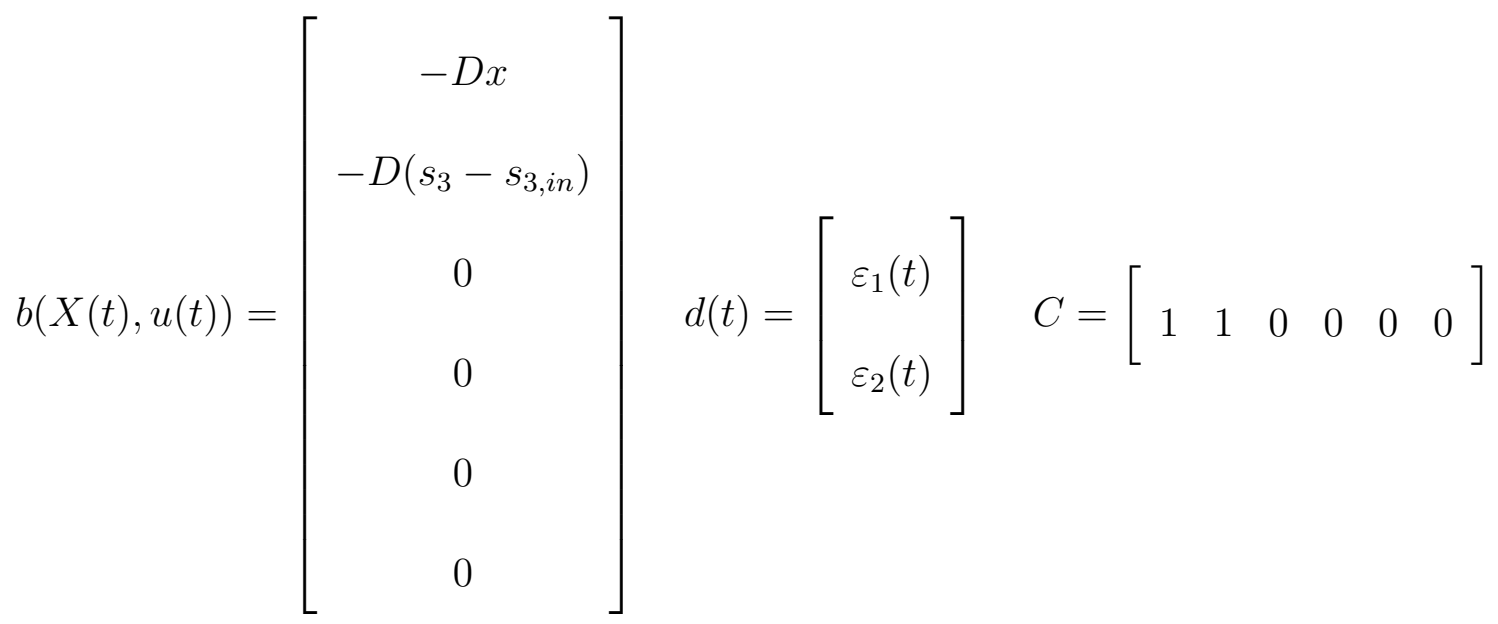

Now applying the theoretical result of section 3, an continuous-discrete observer for system (56) takes the form:

(i) a prediction step at $t \in\left[t_{k}, t_{k+1}[\right.$ given by:

$$
\left\{\begin{array}{l}
\dot{\hat{X}}(t)=A\left(y\left(t_{k}\right), u(t)\right) \hat{X}(t)+b\left(y\left(t_{k}\right), u(t)\right) \\
\dot{S}(t)=-\theta S(t)-A^{T}\left(y\left(t_{k}\right), u(t)\right) S(t)-S(t) A\left(y\left(t_{k}\right), u(t)\right)
\end{array}\right.
$$

(ii) a correction step at $t=t_{k+1}$ given by:

$$
\left\{\begin{array}{l}
\hat{X}\left(t_{k+1}\right)=\hat{X}^{-}\left(t_{k+1}\right)-\rho \delta S^{-1}\left(t_{k+1}\right) C^{T}\left(C \hat{X}^{-}\left(t_{k+1}\right)-y\left(t_{k+1}\right)\right) \\
S\left(t_{k+1}\right)=S^{-}\left(t_{k+1}\right)+\delta C^{T} C
\end{array}\right.
$$


where $\delta$ is the discrete sampling period and $\rho \geq 1$ is a constant.

\subsection{Simulation results}

In order to illustrate the performances of estimators (57.58), we compared the corresponding results with data obtained from simulation, i.e. we simulated the process model (52) by considering kinetic expressions given by (53). The numerical values of system parameters are given in the Table 1.

We suppose that the reactor works in continuous mode, with the dilution rate $D(t)$ varying between $0.1 h^{-1}$ and $0.2 h^{-1}$ as shown in Fig.1.

The simulations were carried out under the followings initial conditions:

$$
\hat{x}^{0}=0.05 \quad \hat{s}_{3}^{0}=0.05 \quad \hat{\alpha}_{1}^{0}=1.5 \quad \hat{\alpha}_{2}^{0}=0.02 \quad \hat{\tilde{\alpha}}_{1}^{0}=0 \quad \hat{\tilde{\alpha}}_{2}^{0}=0
$$

In order to show the effects of the initial estimation of $\alpha_{i}$, the value of $\hat{\alpha}_{i}$ is initialized with an error of 30 compared to the initial simulated value. The sampling period is $\delta=25 \mathrm{~min}$ and the tuning parameter $\theta$ given in equations (58) can have different values. In our case, $\theta=1.56$ and $\rho=3$ give a satisfactory estimation.

The corresponding results are presented in Fig.2 and Fig.3. As can be seen from these figures, the estimator converges after $3 \mathrm{~h}$.

\section{Conclusion}

In this paper, we gave sufficient conditions on input and sampling time which allow to construct an exponential observer for continuous-discrete time state affine systems up to the output injection. The problem of observer synthesis for these systems is related to the input 
excitation and the sampling time of the output measurements. We therefore gave a sufficient condition on the sampling time, which allows to preserve some observability properties: In particular, the asymptotic observability of continuous systems, which is induced by regularly persistent inputs, is preserved for continuous-discrete time systems.

The obtained result is then exploited to estimate reaction rates in the biological denitrification process. These promising results could be used in the future using experimental data and/or to create a control system based on nonlinear control laws that allows us to manipulate the variables that take part in the bioreactor.

\section{References}




\begin{tabular}{llc}
\hline Parameters & Values & Units \\
\hline$\mu_{1 m}$ & 0.18 & $h^{-1}$ \\
$\mu_{2 m}$ & 0.09 & $h^{-1}$ \\
$k_{1}$ & 0.05 & $\mathrm{~g} / 1$ \\
$k_{2}$ & 0.07 & $\mathrm{~g} / 1$ \\
$k_{3}$ & 0.047 & $\mathrm{~g} / \mathrm{l}$ \\
$k_{I}$ & 0.005 & $\mathrm{~g} / \mathrm{l}$ \\
$y_{11}$ & 4.7728 & - \\
$y_{12}$ & 3.343 & - \\
$y_{13}$ & 1.1 & - \\
$y_{22}$ & 1.214 & - \\
$y_{23}$ & 1.6 & \\
\hline
\end{tabular}

Table 1: Yields and kinetic parameters during continuous culture. 


\section{List of Figures}

Fig.1. Time evolution of the process input (the dilution rate) and the process outputs

Fig.2. kinetic reaction $\left(\alpha_{1}\right)$ and its estimate $\left(\hat{\alpha}_{1}\right)$

Fig.3. kinetic reaction $\left(\alpha_{2}\right)$ and its estimate $\left(\hat{\alpha}_{2}\right)$ 


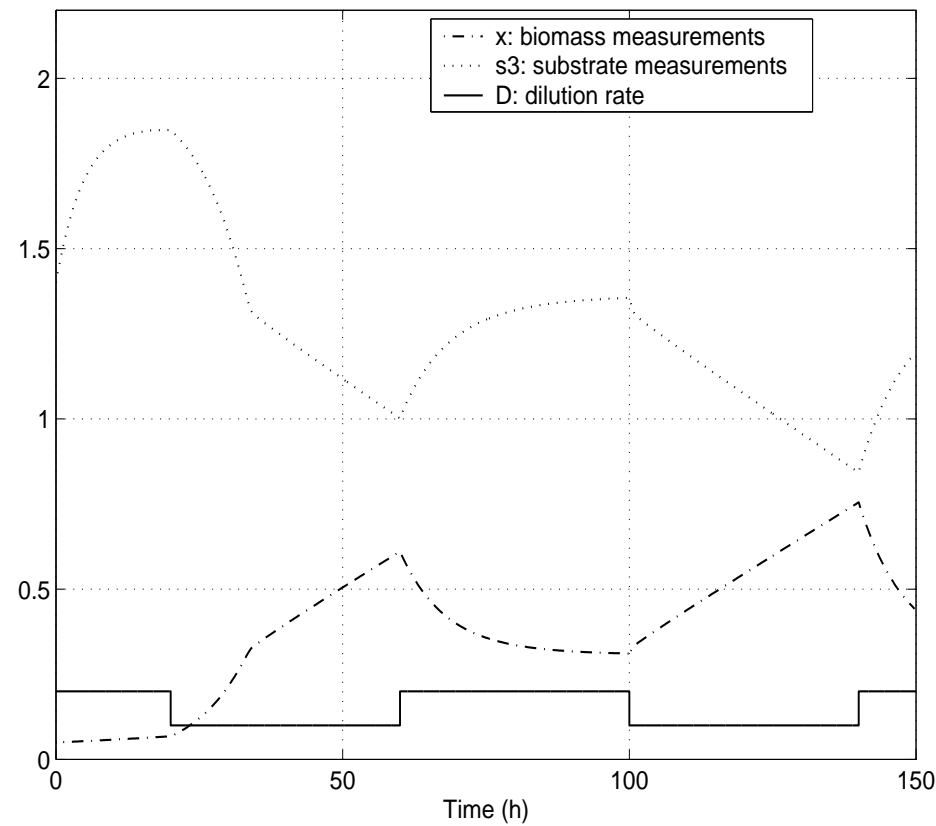

Fig.1. 


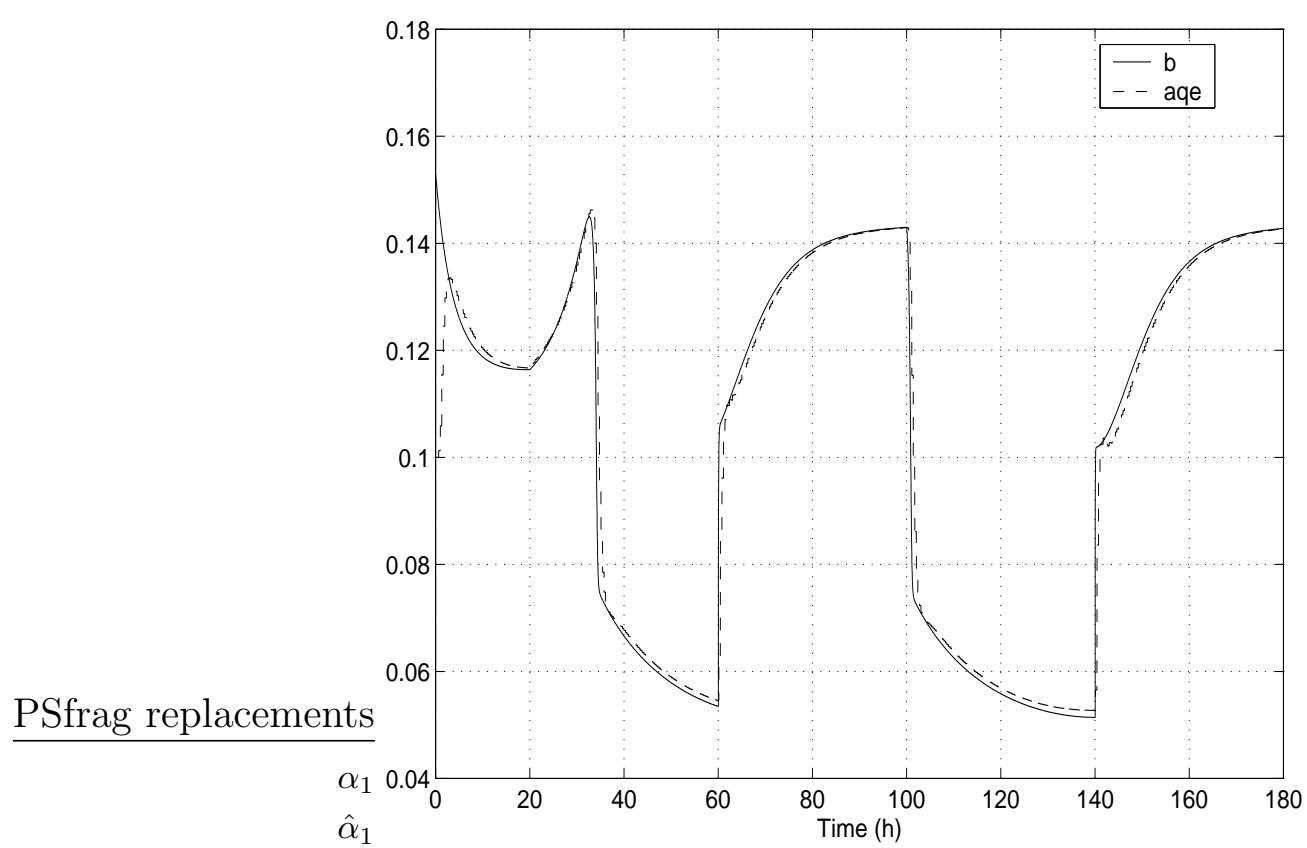

Fig.2. 


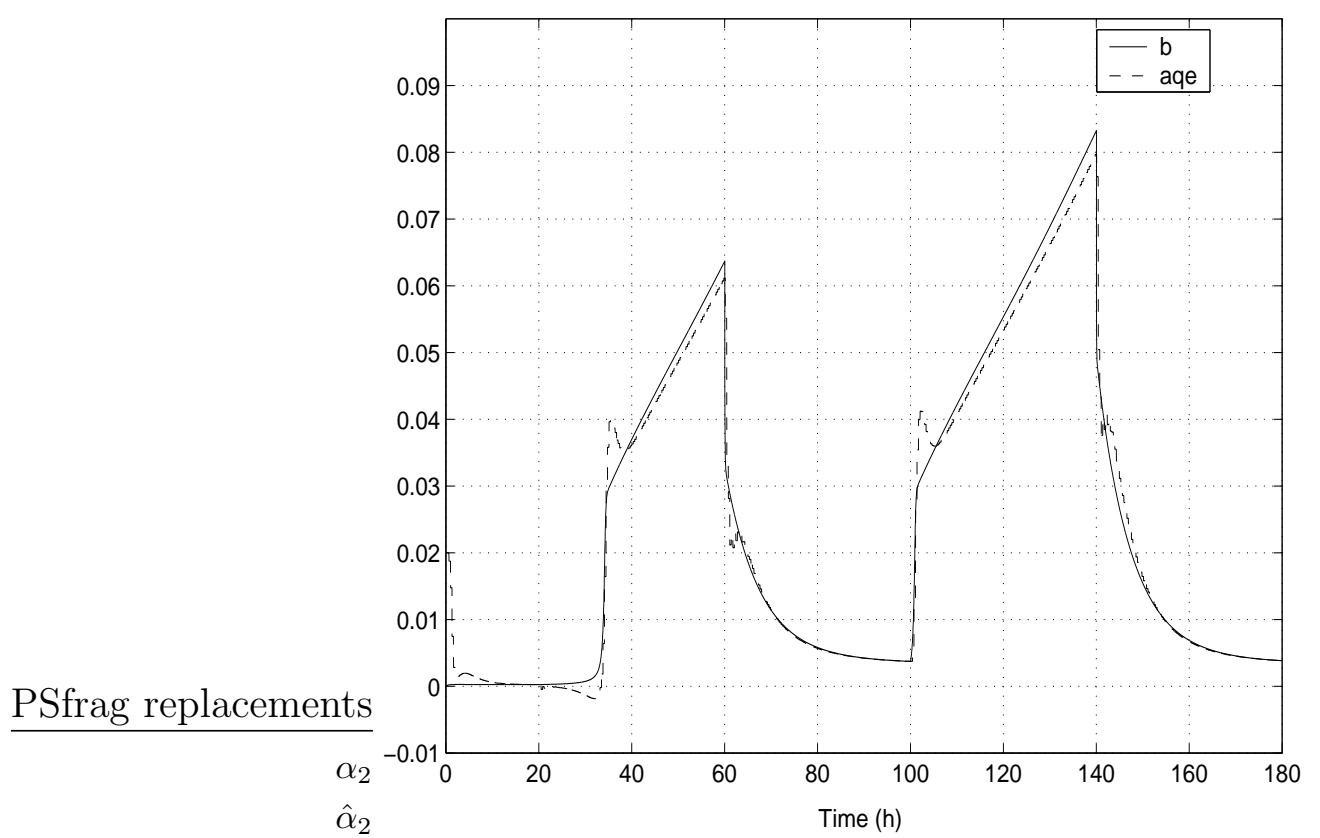

Fig.3. 